\title{
5-fluorouracil encapsulated CS-mPEG nanogels for controlling drug release
}

\author{
Nguyen Dai Hai \\ Institute of Applied Materials Science, Vietnam Academy of Science and Technology \\ Received 29 December 2016; Accepted for publication 28 August 2017
}

\begin{abstract}
The purpose of this study is to synthesize and characterize poly (ethylene glycol) methyl ether (mPEG) conjugated chitosan (CS), mPEG-CS, at different ratios of 5-Fluorouracil (5-FU) delivery (5-FU-loaded mPEG-CS). The chemical cross-linking of these polymers were prepared by using 4-Nitrophenyl chloroformate reagent. The obtained mPEG-CS was characterized by Fourier transform infrared (FTIR) and proton nuclear magnetic resonance $\left({ }^{1} \mathrm{H}\right.$ NMR) spectroscopy. The 5-FU-loaded mPEG-CS particles were nearly spherical in shape with a mean diameter of $61.25 \mathrm{~nm}$, determined by transmission electron microscopy (TEM). In addition, the entrapment efficiency of 5-FU was approximately $10 \%$. Whereas the encapsulation efficiency and loading capacity were independent of different molar ratios of mPEG, there was one factor that particularly stands out, which is 5-FU release behavior. These results indicated that mPEG-CS nanogels present the potential for controlled release of 5-FU working as a delivery system in cancer therapy.
\end{abstract}

Keywords. Poly(ethylene glycol) methyl ether, chitosan, 5-fluorouracil, nanogels, drug delivery system.

\section{INTRODUCTION}

Drug delivery system (DDS) has been an outstanding subject of studying and developing as an original method to control drug release in the blood stream for reducing toxic side-effects in patients. DDS tends to particularly localize and target the drug within desired therapeutic range to expected tissue and cells while maintaining the systemic level of drugs [1]. In order to reach these intentions, nanomaterials (NMs) possess enormous advantages: i) protecting sensitive drug molecules from reticuloendothelial system (RES) in vivo, ii) increasing surface area between loaded drug and tumor tissue, iii) improving the solubility and bioavailability of poorly soluble drugs, iv) and possibility to tailor-making the drug release [2]. Self-assembling nanogels, one of the most effective NMs for ameliorating possibly drug inactivation, have the advantage to straightforward synthesis without the presence of drugs and excellent biocompatibility [3].

In comparison with artificial polymer, chitosan (CS) obtained by deacetylation of chitin has been widely used for the preparation of nanogels due to its unlimited in biocompatibility, biodegradability, hydrophilic polymer, better stability, and low toxicity [4-6]. However, CS is only soluble in aqueous acidic solution $(\mathrm{pH}<6.5)$ because of a lot of amino groups on its chain, which may limit their potential clinical applications [7]. As a result, poly (ethylene glycol) methyl ether (mPEG), an excellent hydrophilic polymer, has been utilized for improving the stability of drug carrier systems in the blood circulation by enhancing water solubility of CS. Besides, grafting mPEG onto CS chain can not only optimize the biocompatibility of CS but also avoid the absorption of protein and evade from RES [812].

In this study, CS and mPEG were conjugated at different molar ratios as controlled-release systems for 5-Fluorouracil (5-FU) delivery, in which CS was designed as cross-linker and mPEG as hydrophilic polymer (mPEG-CS). The obtained nanogels were then characterized by proton nuclear magnetic resonance $\left({ }^{1} \mathrm{H}\right.$ NMR), Fourier transform infrared (FTIR) spectroscopy, and transmission electron microscopy (TEM). Importantly, the loading capacity and release behavior of drug were also evaluated. This study is expected to create a significant opportunity for drug delivery in cancer therapy.

\section{MATERIALS AND METHODS}

\subsection{Materials}

CS (Mw: 100-300 kDa, DDA: $\geq 75 \%$, 5-FU, 
4-nitrophenyl chloroformate (PNC, Mw: 201.56 Da), and poly (ethylene glycol) methyl ether (mPEG, Mw: $5000 \mathrm{Da}$ ) were purchased from SigmaAldrich (St. Louis, MO, USA). All reagents and solvents were used without further purification.

\subsection{Methods}

\subsubsection{Synthesis of $m P E G-P N C$}

The preparation of mPEG-PNC is shown in Scheme 1. Briefly, $0.25 \mathrm{~g}$ of $\mathrm{mPEG}$ was molten under vacuum at $65{ }^{\circ} \mathrm{C}$. Then, $16 \mathrm{mg}$ of PNC was added into the above mPEG solution and the reaction was kept under constant stirring (300 rpm) for $6 \mathrm{~h}$. The mixture was left to cool at $40{ }^{\circ} \mathrm{C}$ for $15 \mathrm{~min}$, followed by adding $5 \mathrm{~mL}$ THF to remove residual unreacted $\mathrm{mPEG}$.

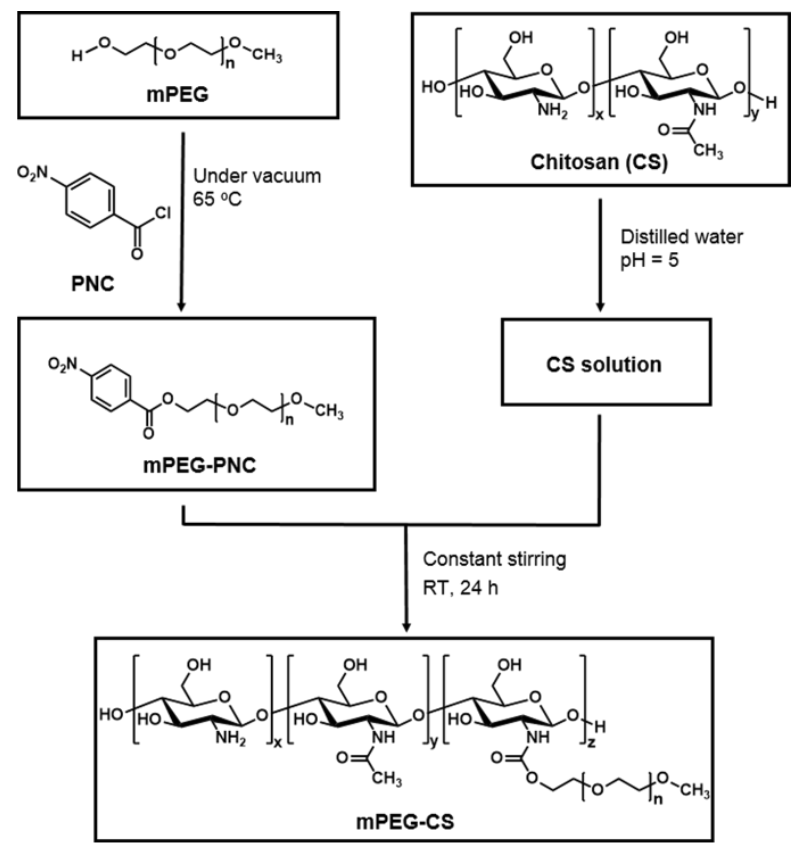

Scheme 1: Synthetic route for mPEG-CS

\subsubsection{Synthesis of $m P E G-C S$}

The CS solution (pH 5) was prepared using $\mathrm{HCl}$ (1M) solution. After that, the mPEG-PNC solution was added drop-wise into the CS solution and the reaction was kept overnight. The mixture was dialyzed against distilled water using Spectra/Por ${ }^{\circledR}$ Dialysis Membrane (MWCO: 12-14 kDa) and then freeze-dried to obtain mPEG-CS conjugate. The sample was prepared at different molar ratios $(1: 1$, 5:1, and 10:1) of mPEG and CS (Scheme 1).

\subsubsection{Drug loading content (DLC) and drug loading} efficiency (DLE)
Initially, $10 \mathrm{mg}$ of 5-FU and $100 \mathrm{mg}$ of mPEG-CS conjugate were converted into liquid by deionized water. The mixture of both solutions was then sonicated for $60 \mathrm{~min}$ and magnetically stirred for 24 $\mathrm{h}$. The solution was later dialyzed against distilled water for removing free-drug. Lastly, the sample was obtained under solid phase by lyophilization.

Three different mPEG-CS samples were dissolved independently with $1 \mathrm{~mL} \mathrm{CH}_{3} \mathrm{COOH}(0.25$ $\mathrm{M})$ and $1 \mathrm{~mL}$ acetonitrile, sonicated for $30 \mathrm{~min}$, and then aliquots were taken out and filtered. These experiments were taken in triplicate for measuring free-5-FU concentration, which were analyzed at $\lambda_{\max }=260 \mathrm{~nm}$ by HPLC method. The \% DLC and \% DLE were calculated using following equations.

DLE $(\%)=($ weight of drug in particles/ weight of drug feed initially) $\times 100$

DLC $(\%)=($ weight of drug in particles/ weight of particles and drug $) \times 100$

\subsubsection{In vitro release}

The release behavior of 5-FU nanogels was carried out in phosphate buffer saline (PBS, $137 \mathrm{mmol} / \mathrm{L}$ $\mathrm{NaCl} ; 2.7 \mathrm{mmol} / \mathrm{L} \mathrm{KCl} ; 10 \mathrm{mmol} / \mathrm{L} \mathrm{Na} \mathrm{NPO}_{4} ; 1.8$ $\left.\mathrm{mmol} / \mathrm{L}_{\mathrm{KH}_{2}} \mathrm{PO}_{4}\right)$ and Tween $80(2 \% \mathrm{v} / \mathrm{v})$ by dialysis method. 5-FU-loaded nanogels solution (25 $\mathrm{mg} / \mathrm{mL})$ was added into a dialysis bag $(\mathrm{MWCO}=3.5$ $\mathrm{kDa}$ ) which was submerged fully into $12.5 \mathrm{~mL}$ of the medium at $37{ }^{\circ} \mathrm{C}$ with constant stirring for $12 \mathrm{~h}$. At appropriate time intervals $(2,4,6,8,10,12 \mathrm{~h})$, $0.5 \mathrm{~mL}$ aliquots were withdrawn from the system and then filtered with $0.20 \mu \mathrm{m}$ millipore filter. This experiment were repeated three times as described above for HPLC analysis.

\section{RESULTS AND DISCUSSION}

\subsection{Characterization}

The mPEG-CS was characterized by ${ }^{1} \mathrm{H}$ NMR analysis (Fig. 1). The solvent peak of $\mathrm{D}_{2} \mathrm{O}$ was found in $4.74 \mathrm{ppm}$. The typical peak at $3.4-3.94 \mathrm{ppm}$ (H-b, H-c, H-d, H-e, H-2, and $\mathrm{H}-3$ ) was assigned to methylene protons of CS saccharide units and repeat units in mPEG. Peaks at $2.95 \mathrm{ppm}(\mathrm{H}-\mathrm{c})$ and 3.37 ppm (H-1) were attributed to - CH-NH- from CS and $-\mathrm{OCH}_{3}$ from mPEG, respectively. The peak at 1.95$1.98 \mathrm{ppm}(\mathrm{H}-\mathrm{f})$ was assigned to $-\mathrm{NH}-\mathrm{CO}-\mathrm{CH}_{3}$. These results demonstrated that mPEG was successfully conjugated to the surface of CS.

The FTIR spectra of mPEG-CS (i), CS (ii), and mPEG (iii) are presented in Fig. 2. The spectrum of CS reveals distinctive absorption bands at $1656 \mathrm{~cm}^{-1}$ 
(amide I), $1594 \mathrm{~cm}^{-1}$ (-NH 2 bending), and $1400 \mathrm{~cm}^{-1}$ (amide III) (Fig. 2ii). The absorption bands at 1150 $\mathrm{cm}^{-1}$ (asymmetric stretching of the COOOC bridge), 1092 , and $1042 \mathrm{~cm}^{-1}$ (skeletal vibration involving the COO stretching) were assigned to its saccharine structure. In addition, the increased intensity of the peaks at around 2910 and 2830, and $1094 \mathrm{~cm}^{-1}$ show the $\mathrm{CH}_{2}$ groups, and $\mathrm{C}-\mathrm{O}-\mathrm{C}$ stretch of mPEG, respectively (Fig. 2iii). As shown in Fig. 2i, the CS amide peaks slightly shifted to $1631 \mathrm{~cm}^{-1}$ and 1529 $\mathrm{cm}^{-1}$, respectively. The shifts were possibly due to hydrogen bonding between amide carbonyl with mPEG hydroxyl. The increased intensity of the peaks at around $2924 \mathrm{~cm}^{-1}$ and $1100 \mathrm{~cm}^{-1}$ indicated the $\mathrm{CH}_{2}$ groups and $\mathrm{C}-\mathrm{O}-\mathrm{C}$ stretch of mPEG, respectively. These results indicated that $\mathrm{mPEG}-\mathrm{CS}$ conjugate was prepared.

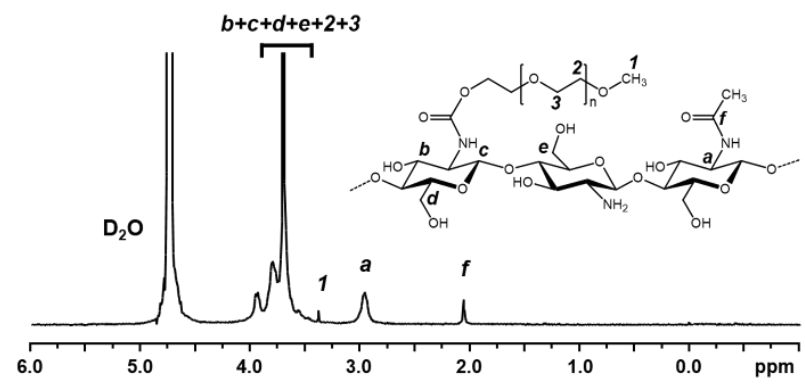

Figure 1: ${ }^{1} \mathrm{H}$ NMR spectrum of mPEG-CS

The particle size of mPEG-CS nanogels was found to be nearly spherical in shape (Fig. 3a) with an average diameter of $61.25 \mathrm{~nm}$ (Fig. 3b). According to previous reports, nanoparticles are ranging from $20 \mathrm{~nm}$ to $100 \mathrm{~nm}$ in size that are preferred to accumulate in solid tumors caused by the enhanced permeability and retention (EPR) effect.

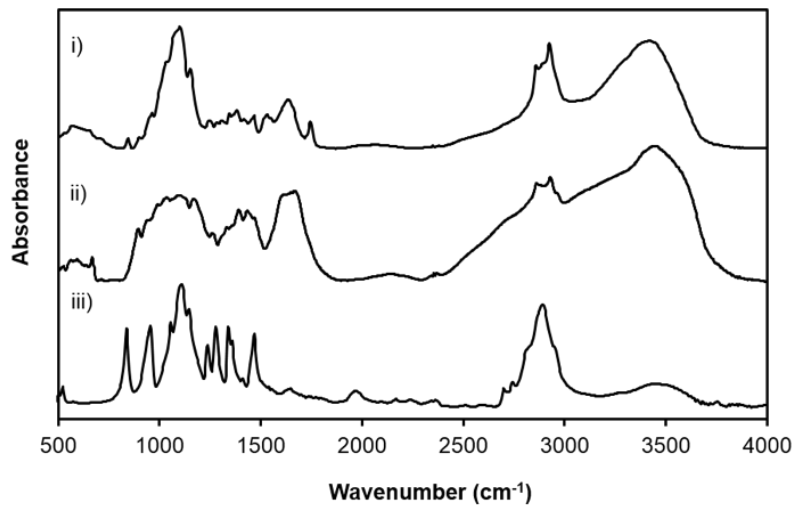

Figure 2: FTIR spectra of (i) mPEG-CS, (ii) CS and (iii) $\mathrm{mPEG}$
The small size allows prolonged circulation half-life of the drug, providing more opportunities for nanoparticles to accumulate at the target site. Therefore, 5-FU-loaded mPEG-CS nanogels might be suitable for drug delivery.

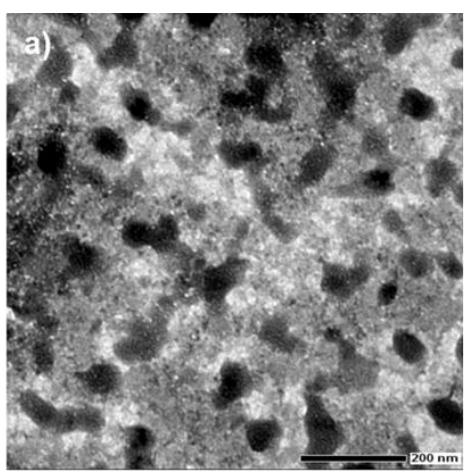

b)

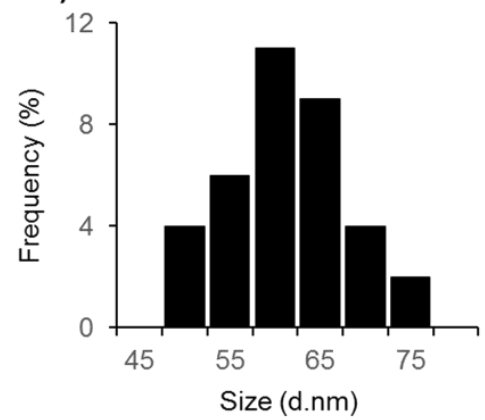

Figure 3: (a) TEM image and (b) particle size distribution of 5-FU-loaded mPEG-CS nanogels

\subsection{Drug loading and release behavior}

The DLE of 5-FU-loaded mPEG-CS nanogels were $11.5 \%$ at mPEG-CS (1:1), $10.0 \%$ at mPEG-CS (5:1), and $10.39 \%$ at mPEG-CS (10:1). There were no significant differences of 5-FU loading among mPEG-CS ratios, which means DLE and DLC of the drug did not depend on different quantifications of mPEG over CS.

The in vitro release behavior of 5-FU was carried out in PBS (pH 7.4) at $37{ }^{\circ} \mathrm{C}$ (Fig. 4). The release of 5-FU from mPEG-CS nanogels $(1: 1)$ was $23 \%$ at 2 $\mathrm{h}$, and then increased to $39 \%$ at $4 \mathrm{~h}$. The release of drug was later relatively slow up to $12 \mathrm{~h}(41 \%)$, only $2 \% 5$-FU was released during that period of time. The release of 5-FU released from MPEG-CS (5:1) was $17 \%$ at $2 \mathrm{~h}$ and $33 \%$ at $4 \mathrm{~h}$. After $12 \mathrm{~h}, 37$ $\%$ 5-FU was slowly delivered. In comparison with mPEG-CS (10:1), the drug was rapidly freed up 94\% at $12 \mathrm{~h}$. Based on these results, mPEG-CS (1:1) and mPEG-CS (5:1) were suitable for prolonged release, rather than mPEG-CS (10:1), the highest performance $\mathrm{mPEG}$. 


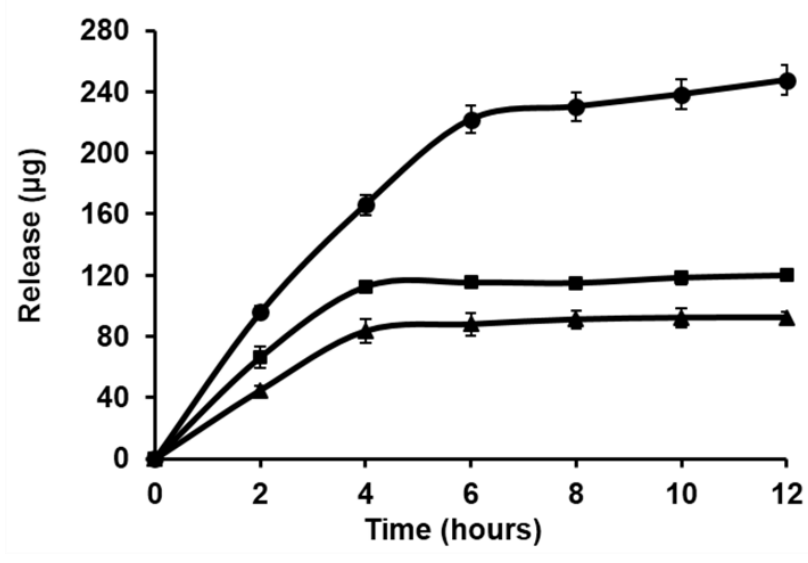

Figure 4: Drug release of mPEG-CS at different ratios Square:mPEG-CS (1:1); triangular:mPEG-CS (5:1); circle:mPEG-CS $(10: 1)$

\section{CONCLUSION}

According to the results of ${ }^{1} \mathrm{H}$ NMR and FTIR analysis, 5-FU-loaded mPEG-CS nanogels were successfully synthesized. The particle size of nanogels was nearly spherical in shape with an average diameter of $61.25 \mathrm{~nm}$. In addition, 5-FU was relatively loaded into $\mathrm{mPEG}-\mathrm{CS}$ nanogels and the release behavior of 5-FU showed that mPEG-CS (1:1) and mPEG-CS (5:1) have great potential as controlled delivery system in cancer therapy.

Acknowledgment. This research was supported by Institute of Applied Materials Science, Vietnam Academy of Science and Technology.

\section{REFERENCES}

1. M. Goldberg, R. Langer, X. Jia. Nanostructured materials for applications in drug delivery and tissue engineering, J. Biomater. Sci. Polymer. Ed., 18(3), 241-268 (2007).

2. O. C. Farokhzad, R. Langer. Impact of nanotechnology on drug delivery, ACS. Nano., 3, 16-20 (2009).

3. Elena V. Batrakova, Alexander V. Kabanov. Pluronic block copolymers: evaluation of drug delivery concept from inert nanocarriers to biological response modifier, J. Control Release, 130, 98-106 (2008).

4. A. A. Sunil, N. M. Nadagouda, M. A. Tejraj. Recent advances on chitosan-based micro- and nanoparticles in drug delivery, J. Control. Release, 100, 5-28 (2004).

5. Hoang D. Q., Tran T. V., Tran N. Q., Nguyen C. K., Nguyen T. H., Truong M. D., Tran D. L., Thu L. V. and Nguyen D. H. Functionalization of $\mathrm{Fe} \mathrm{O} 4$ nanoparticles with biodegradable chitosan-graftedmPEG for paclitaxel delivery, Green Processing and Synthesis, Green Processing and Synthesis, 5, 459466 (2016).

6. Hoang Phuc D., Thi Hiep N., Ngoc Phuc Chau D., Thi Thu Hoai N., Chan Khon H., Van Toi V., Dai Hai N. and Chi Bao B. Fabrication of HyaluronanPoly (vinylphosphonic acid)-Chitosan Hydrogel for Wound Healing Application, International Journal of Polymer Science, 2016 (2016).

7. P. Chan, M. Kurisawa, J. E. Chung et al. Synthesis and characterization of chitosan-339 g-poly (ethylene glycol)-folate as a non-viral carrier for tumor-targeted gene delivery, Biomaterials., $\mathbf{2 8}$, 540-549 (2007).

8. S. Y. Zhu, F. Qian, Y. Zhang et al. Synthesis and characterization of PEG modified 345 $N$-trimethylaminoethylmethacrylate CS nanoparticle, Eur. Polym. J., 43, 2244-2253 (2007).

9. A. R. Kulkarni, Y. H. Lin, H. F. Liang et al. A novel method for the preparation of nanoaggregates of methoxy polyethyleneglycol linked chitosan, J. Nanosci. Nanotechnol., 6, 2867-2873 (2006).

10. Nguyen D. H., Lee J. S., Bae J. W., Choi J. H., Lee Y., Son J. Y. and Park K. D. Targeted doxorubicin nanotherapy strongly suppressing growth of multidrug resistant tumor in mice, International journal of pharmaceutics, 495, 329-335 (2015).

11. Nguyen D. H., Lee J. S., Choi J. H., Lee Y., Son J. Y., Bae J. W.,Lee K. and Park K. D. Heparin nanogel-containing liposomes for intracellular RNase delivery, Macromolecular Research, 23, 765769 (2015).

12. Nguyen D. H., Tran N. Q. and Nguyen C. K. Tetronic-grafted chitosan hydrogel as an injectable and biocompatible scaffold for biomedical applications, Journal of Biomaterials Science, Polymer Edition, 24, 1636-1648 (2013).

\section{Corresponding author: Nguyen Dai Hai}

Department of Biomaterials \& Bioengineering

Institute of Applied Materials Science, VAST

01 TL29, District 12, Ho Chi Minh City

E-mail: nguyendaihai @iams.vast.vn;

Telephone: +84-(08)3-8298987 / 939866778 (Off). 